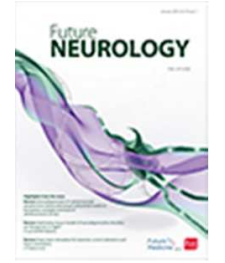

\title{
Protective Effect or Missed Diagnosis? Females with Autism Spectrum Disorder
}

\begin{tabular}{|r|l|}
\hline Journal: & Future Neurology \\
\hline Manuscript ID & FNL-2017-0006.R1 \\
\hline Manuscript Type: & Review \\
\hline Keywords: & Autism, Asperger's, Generalized anxiety disorder \\
\hline \multicolumn{2}{|l}{} \\
\hline
\end{tabular}

\section{SCHOLARONE ${ }^{\text {m }}$ \\ Manuscripts}




\begin{abstract}
Autism Spectrum Disorder (ASD) has historically been diagnosed more frequently in males than females. One explanation for this is the 'female protective effect': that there is something inherent in being female that makes girls and women less susceptible to ASD. Another possibility is that ASD is under-diagnosed in girls and women, due to the existence of a 'female autism phenotype', which is not well captured by current, male-biased diagnostic criteria. To evaluate the 'female protective effect' and 'female autism phenotype' hypotheses, this narrative review describes recent developments exploring the genetic underpinning and behavioural expression of ASD in females. We then look at ways to better identify females with ASD who may be missed under the current diagnostic criteria.
\end{abstract}

\title{
Keywords
}

Autism, Autism Spectrum Disorders, Gender Differences, Sex Differences, Genetics, Diagnosis, Person-Environment Fit

\section{Introduction}

Autism Spectrum Disorder (ASD) is a group of neurodevelopmental conditions characterised by deficits in social communication and reciprocity, and by restricted and repetitive interests and behaviours ${ }^{1}$. ASD is the current term used to integrate the previously separate diagnostic categories of autistic disorder, Asperger's disorder, childhood disintegrative disorder and pervasive developmental disorder - not otherwise specified (aka 'atypical autism' [2]). ASD is a lifelong condition that is present from birth. Although adult diagnosis is possible, symptoms must be reported as present from early developmental stages in order for the

\footnotetext{
${ }^{1}$ In line with preferences from a majority of the autism community [1], we use identity-first language (e.g. 'autistic person') to refer to individuals with an ASD throughout this paper, while recognising that others might prefer person-first language (e.g. 'person with autism').
} 
condition to be diagnosed. Around one third of individuals with ASD also have intellectual disability [3]: the full autism spectrum includes individuals of a wide range of abilities.

\section{Gender differences in diagnosis}

ASD affects approximately one to two per cent of the population [3, 4], and is much more likely to be diagnosed in males than in females. Gender ratios in diagnosis vary depending on the country, cohort, and characteristics of those included, but average ratios are reported at around four males for every one female [3-5]. However, when looking at those individuals with intellectual disability, the male-to-female ratio narrows significantly to around two-toone [6]. In addition, population-based studies, which screen all participants in order to identify cases regardless of whether they have already been diagnosed by local services, suggest that the male-to-female ratio is closer to $3: 1[7,8]$. This is in contrast to the average of around 4-5:1 generally reported in studies based on clinically-diagnosed cases [9]. The fact that a lower proportion of females are found in clinical samples than in community-based, epidemiological samples suggests that there is a bias against autistic girls and women coming to clinical attention. This bias may have existed from the very first conceptions of ASD; only 3 of 11 children in Kanner's original cases were female, and no girls were included in Asperger's first four cases [10].

Those seeking to understand the preponderance of males diagnosed with ASD have pursued two contrasting explanations. The first is that there is something about being female which inherently protects the majority of girls and women from ASD - the 'Female Protective Effect' [11]. This idea assumes that the observed gender ratio reflects reality, and that males really are three to four times more likely to have ASD than females. The second is that the current gender ratio reflects an underestimation of the true prevalence of autistic females, due in part to gender differences in the behavioural expression of ASD and male-biased 
diagnostic criteria [6]. This explanation suggests that significant numbers of autistic females are currently being missed by clinical services and hence the true female prevalence of ASD is still unknown. These explanations are not mutually exclusive; we will argue that a combination of both is likely to explain both the higher male diagnostic rate and the increasing numbers of females diagnosed with ASD in recent years.

This article will examine evidence surrounding both of these explanations, starting with some of the evidence for a protective female genetic factor, and then moving to look at how differences in the behavioural expression of ASD may lead females to be missed by clinical services. We will then explore further reasons why females who do have ASD may go undiagnosed, including the influence of co-morbid conditions which may mask autistic females' ASD. We finish by looking at ways in which future research can contribute to both of these explanations in order to improve our understanding of ASD and our ability to identify those who require services and support.

\section{The Female Protective Effect: Genetic and environmental risk}

ASD shows higher concordance between monozygotic than dizygotic twins [12], with its heritability estimated to be between 64 - 91\% [13]. The effect of commonly shared genetic variance has the greatest impact on whether or not an individual develops ASD, with $49 \%$ of variance explained by the cumulative effect of multiple mutations found commonly across the general population [14]. This additive effect is the result of many genetic mutations all contributing to the overall expression of the condition, rather than being dependent on a single gene or specific interaction to produce ASD [15]. In contrast, rarely occurring mutations of large individual effect were found to contribute only $3 \%$ of variance [14]. Several de novo (non-inherited) mutations, such as those in the SHANK gene family [16] and 
the NRXN gene family [17], have been associated with ASD. However, mutations in these areas are also associated with other developmental disorders and so do not necessarily represent a unique genetic pathway to $\operatorname{ASD}[18,19]$. A polygenic model with multiple additive genetic effects is therefore proposed as a better explanation of heritability than single gene mutation or non-additive, interaction-dependent genetic effects [20]. It has also been suggested that ASD may in fact be a collection of conditions with different genetic causes and similar behavioural expressions [21].

Genetic risk factors for ASD are likely to interact with environmental risk factors, including pre- and post-natal factors such as maternal age, socioeconomic status, and in-vitro exposure to medications and toxins $(22,23]$. While many studies of these environmental factors are based on animal models or are underpowered to detect risk factors across entire populations [22], there is undoubtedly a substantial impact of the environment on both shared autism variance (e.g. twin symptom concordance) and population-wide autism risk [24]. This review does not have the space to describe the many environmental factors thought to contribute to ASD, or how they may interact with (and be influenced by) genetic factors, but we direct interested readers to a recent review of the literature for more information [23].

\section{Female Protective Effect}

The Female Protective Effect (FPE) theory suggests that females are relatively protected from ASD compared to males, such that they require a higher degree of genetic and environmental risk to express the symptoms of ASD [11]. In other words, this idea proposes that the degree of aetiological risk sufficient to produce behavioural expression of ASD in males does not produce the same level of impairment in females. There are two main sources of genetic evidence for this argument: the study of non-inherited, de novo mutations, and studies comparing occurrence of ASD amongst relatives of male and female probands with ASD. 
Comparisons of males' and females' genetic predispositions to ASD offer some support for the suggestion that females may be relatively protected from ASD. Several studies have shown that autistic females possess more de novo mutations than males with equivalent severity of symptoms $[25,26]$. One interpretation of such findings is that for females, in comparison to males, a larger genetic hit is required for the full ASD phenotype to be expressed. In other words, there must be a resilience factor shared by most females, which 'protects' them from behaviourally expressing ASD even when they carry genetic risk for the condition. This would explain why females are less commonly diagnosed with ASD than males; the threshold at which genetic mutation produces behavioural symptoms is higher in females than in males. The precise nature of this putative female protective effect has not yet been identified, although it may be related to the $\mathrm{X}$ chromosome and/or to the role of sex hormones such as androgens [27- 29].

Additional support for the female protective effect has been sought in studies looking at the genetic relatives of those diagnosed with ASD. The logic runs as follows: if females are protected against autism risk, then autistic females should, on average, carry greater genetic risk for autism than males; and given that most genetic risk for autism is inherited, blood relatives of autistic females should, on average, carry more genetic risk of ASD than those of autistic males. Therefore the prediction is that female autistic probands should have more autistic relatives than do male autistic probands. There is some evidence in support of this. Several studies have shown that first-degree relatives of autistic females have more ASD symptoms than first-degree relatives of autistic males [30-32]. Nevertheless, these findings have been contradicted, with one study showing no greater likelihood of ASD amongst relatives of autistic females [33]. Further research, meta-analysis, and the examination of a range of genetic and behavioural profiles are needed to identify how much influence genetic variation has on the diagnosis of males and females with ASD. 
Some environmental factors may also contribute to the female protective effect [23]. Inuterine exposure to SSRIs [34] and steroids including progesterone and testosterone [35] are associated with greater autism likelihood amongst male children than female. Some evidence suggests higher maternal age at conception may increase likelihood of autism amongst males, but not females [36]. These factors are likely to interact with any familial or de novo genetic risks, further increasing the likelihood of autism in males and thus offering relatively greater protection in females.

It should be noted that studies of gender differences in susceptibility to genetic and environmental risk rely on the assumption that current case ascertainment methods (i.e., diagnostic criteria and mechanisms for applying these) are not biased against either sex. For example, if case ascertainment were biased against females, the finding that diagnosed autistic females carry greater genetic risk becomes difficult to interpret: perhaps it arises because there are autistic females with lower genetic risk who were excluded from studies as they were not picked up clinically. As we discuss below, such a situation is a distinct possibility, which undermines current evidence for the female protective effect theory.

\section{The Female Phenotype: Behaviour}

An alternative explanation for gender differences in diagnostic rates is that ASD is expressed differently in females and males. As current diagnostic criteria rely on behavioural observations to identify ASD, any behavioural expression of ASD which does not meet these criteria will not result in an ASD diagnosis. It is proposed that autistic females may express their impairments in different ways to autistic males, and so slip 'under the radar' during diagnostic assessments [37]. The lower rate of ASD in females is therefore proposed partially to reflect a greater proportion of cases being missed or misdiagnosed [38]. This argument is 
based on the presupposition that there can be both environmental and genetic contributing factors, which may produce gender-specific behavioural expressions of ASD in females compared to males [39].

\section{Gender differences in presentation}

Most research into autism gender differences has compared autistic males and females, recruited from clinical samples, in terms of their core autism symptoms. Such work has mostly found that males and females attending autism clinics do not differ in terms of their autistic social and communication impairments, as measured by current, standardised instruments $[28,40,41]$. By contrast, there is clear evidence that in these clinical samples autistic females have fewer restricted and repetitive behaviours/interests than autistic males $[6,42]$. One issue is that there is significant variation in IQ, age, language ability and other characteristics between different samples, and therefore it is difficult to draw comparisons across different studies [6].

While some research has shown that autistic females may have more severe impairments than males, recent studies have found that females' lower IQ completely mediated this difference [43]: when autistic individuals are matched on IQ, the level of impairment is the same or even reduced amongst females [44]. However, as females diagnosed with ASD are more likely to have intellectual disability than males [45], the overall clinical presentation of female ASD is of greater impairment. This raises the question of whether higher IQ is protective of autism in females, or whether there are females with autism and typical IQ who are currently being missed. If these females' symptoms are the same as males with ASD, they should be identified in similar proportions to females with low IQ. However, male-to-female ratios are substantially higher at the high-cognitive-ability end of the spectrum. 
Recent studies have found that males are more likely to receive a full ASD diagnosis, whereas females are more likely to receive partial diagnoses of related, less specific disorders such as Pervasive Developmental Disorder [46] or the newly introduced Social Communication Disorder [2]. These reflect general perceptions that it is 'harder' to diagnose autistic girls and women - they are less likely to fit traditional diagnostic criteria than males, even though adaptive and functional difficulties are obviously present [45, 47]. Current goldstandard behavioural diagnostic assessments such as the Autism Diagnostic Observation Schedule (ADOS; 48) were developed using predominantly male samples [49], and have been found to pick up males more reliably, missing a disproportionate number of females with ASD [50]. This supports arguments that these behavioural assessments only identify those females who display male-typical behaviours [6].

Autistic females also show variations in other characteristics when compared to males. For example, females have been found to show poorer executive function on a variety of measures compared to males [51] although other studies have shown no gender differences or even a female advantage $[52,53]$. Autistic females have also been shown to perform better on theory of mind tasks than males [54], and to have more typical play styles and closer friendships $[55,56]$. Studies of children have generally shown that autistic boys display more externalising behaviours, including inattention and hyperactivity, than girls [57], whereas girls may be more likely to display internalising behaviours such as anxiety than boys [58]. However, these results vary across ages, and there is little research into gender differences in these characteristics beyond adolescence.

\section{Camouflaging by females}

Another component of the female phenotype which in itself may lead to lack of ASD diagnosis amongst females, is the development of camouflaging or compensatory behaviours 
$[53,59]$. Some of the strengths identified as characteristic of autistic females may enable them to compensate for ASD impairments, such as better cognitive flexibility which may allow autistic girls and women to select learned social strategies more readily than males [60]. Simultaneously, several self-reported anecdotes and a few newly emerging studies have identified camouflaging techniques used by autistic females to mask and distract from their ASD difficulties, and to present a more socially competent self than they actually feel [61, 62]. These strategies, which are used both to cope with ASD difficulties and minimise others' awareness of them, may result in under-identification of females who are struggling with their ASD, as the outward portrayal to teachers or clinicians is of someone who is developing typically $[59,63]$. This behavioural technique may also explain why increasing numbers of females receive their ASD diagnosis in adolescence or adulthood, when either the effort of maintaining camouflaging strategies or the ASD itself becomes overwhelming, and so brings the individual to clinical attention [62]. Interestingly, camouflaging strategies are also reported by autistic males, although gender differences in the success of and satisfaction with camouflaging may still exist [64].

The female behavioural expression of ASD is therefore qualitatively different to the male expression in at least some key areas, and this variation may lead to under-recognition of ASD amongst females. Autistic females appear to require greater impairments across a range of areas, including core and associated characteristics of ASD, in order to receive an ASD diagnosis, when compared to males [45], and therefore females with lesser impairments are more likely to be missed by clinical services [7]. This is in contrast to other neurodevelopmental conditions more commonly found in males than females such as ADHD, where females appear to express similar levels of impairment to males when assessed regardless of previous referral [65]. However females with ADHD who are referred to 
clinical services tend to have lower levels of impairment than males, suggesting that gendered referral biases are present across neurodevelopmental disorders [66].

Greater emphasis within ASD diagnostic criteria on the traditionally male impairments and strengths, such as stereotyping 'typical' ASD interests like trains which are more commonly shown by males [46], may lead to diagnostic bias where only those females who best meet these criteria are identified. Any research assessing gender differences in ASD is therefore limited by basing its female sample on those who appear most 'typically' autistic, and this raises the possibility of the existence of a group of females who have ASD but do not display it in the typical ways, and so are not being identified clinically.

\section{Missing Women: Co-occurring conditions and environmental fit}

The evidence presented so far suggests that there are a significant number of autistic females who are not being picked up by current ASD assessments, leading to a skewed perception of gender ratios in ASD prevalence. However, population-based prevalence studies have shown a decrease in the male:female diagnostic ratio in recent years [41], suggesting that the proportion of clinically-identified autistic females may be increasing over time.

This may reflect a broadening of diagnostic criteria over time so that those individuals with more subtle presentations of ASD are more likely to be identified $[67,68]$. Despite this, males are still significantly more likely to be diagnosed with ASD than females, suggesting that there is some sort of male risk and/or female protective component to ASD even when gender-specific behavioural presentations are taken into account. This final section will explore reasons why autistic females of all ages may not be identified despite changing perceptions of ASD presentation, and look at the ways in which females with ASD may come to clinical attention for other conditions. 


\section{Changing environments}

Changes over time, in both the characteristics and abilities of females with ASD, and the environment around them, may contribute to a missed diagnosis. The social deficits of autistic girls are more likely to be missed than those of boys in school environments [42]; autistic girls have been found to fit in better to school social settings such as the playground by appearing to have typical friendships and interactions at first glance, whereas boys are more obviously identified as struggling socially [63]. However, closer examination reveals autistic girls may have significant difficulties maintaining friendships and dealing with conflict, and therefore are not functioning socially to the same extent as their typically developing peers [56]. As previously described, both intuitive and explicit camouflaging techniques mean that adolescent girls can mask their ASD characteristics from others around them, even in clinical settings [62]. Autistic girls and adolescents may therefore miss out on official diagnosis for years due to lack of awareness of their difficulties from others around them. It may however be difficult to maintain this camouflaging as girls get older, as evidenced by reduced gender ratios in clinics assessing adults in comparison to children or adolescents [69] - females may be more easily identified as they get older and their camouflaging strategies fail.

The broadening of diagnostic criteria in recent years may account for the increase in adult diagnosis with ASD and no intellectual disability, particularly for women, which has been observed [70]. As autistic children these individuals would not have met the stricter criteria for ASD diagnosis, and so may have received other diagnoses or simply gone unidentified during their childhood and into adulthood. It is suggested that while some of these individuals may have developed sufficient coping or compensation strategies that their ASD did not adversely affect their everyday lives, others may be superficially masking underlying difficulties which negatively impact them over time [68]. For instance, autistic women report 
lower levels of educational attainment and employment than non-autistic women, and attribute this gap partly to their ASD characteristics holding them back [70]. Of even greater concern are the high levels of sexual assault and other types of abuse reported by women with ASD and high autism traits $[62,71]$. If these assaults are occurring in women and girls with ASD diagnoses, it is likely that similar experiences occur for females with ASD but no diagnosis, who may be less likely to seek support as they are not already known to clinical services.

\section{Co-occurring Conditions}

Up to $70 \%$ of autistic individuals have at least one other mental health condition during their lifetime, a significantly higher proportion than in the general population, with the most common co-morbidities being anxiety and depression $[72,73]$. However, it is difficult to identify the relationship between ASD and other conditions; whether both are caused by the same underlying factors, whether other mental health conditions are a result of living with ASD, or whether these are unrelated but interact to impact a person's quality of life. Recent research in this area provides some suggestions as to the 'lost generation' of autistic females without intellectual disability [68], whose co-morbid diagnoses may serve to hide their underlying ASD. We will focus in particular on three conditions which share some similar characteristics to ASD, but with higher prevalence in females. Evidence for the overlap between ASD and these conditions is, in many cases, still at very early stages, but provides important hypotheses for future research and clinical consideration.

\section{Anorexia Nervosa}

The first condition is anorexia nervosa (AN), which, along with other eating disorders, is diagnosed at a significantly higher rate in females than in males [74]. Individuals with AN are also significantly more likely to have ASD symptoms than the general population [75]. For 
instance, emotional dysregulation and difficulties with set-shifting are associated with both ASD and AN, and may be shared factors affecting the different presentations in these two conditions $[76,77]$. However, it is important to bear in mind that similar cognitive biases have been found in otherwise healthy individuals experiencing starvation [78], therefore it is possible that the similarities between ASD and AN are a temporary result of the eating disorder, rather than a shared underlying cause. Case studies suggest that some women with AN may in fact have an undiagnosed ASD, with self-reported ASD symptoms, based on descriptions of behaviour from before the development of their eating disorder, reaching levels sufficient for an ASD diagnosis [79]. One potential outcome of this research is that females identified as having eating disorders should also be screened for high autism traits, as the co-occurrence of undiagnosed ASD may impact both their general functioning and the most effective treatments for their eating disorder [80].

\section{Anxiety}

The co-occurrence of ASD and anxiety problems has long been demonstrated, with generalised anxiety disorder (GAD) a common response to the unpredictable, often stressful nature of life with ASD [81]. Social anxiety and ASD also share many similar aspects of presentation, and it is suggested that some of the avoidance techniques used by autistic individuals to minimise social difficulties may be misinterpreted as demonstrations of social anxiety [68]. Anxiety problems are also more commonly found in females with and without ASD $[82,83]$, although are still highly prevalent amongst males [84]. It has been suggested that anxiety disorders may 'mask' some ASD symptoms in women, as the anxiety may be the most pressing clinical concern and so underlying ASD difficulties remain unidentified [68, 85]. Indeed, autistic women who were diagnosed in adulthood report high levels of anxiety and diagnoses of anxiety disorders from adolescence, and often first present clinically in mental health services, seeking help for their anxiety problems [61]. Women with anxiety 
FEMALES WITH AUTISM SPECTRUM DISORDER

disorders again provide a potential screening group to identify some of the 'missing' autistic women who are not currently being identified.

\section{Gender Dysphoria}

A final condition with higher than typical co-occurrence with ASD is gender dysphoria (GD), also known as Gender Identity Disorder. GD is the term used to describe individuals who do not identify with the gender they have been assigned from birth; this can be expressed through a desire to transition into the identity of another gender, or to identify as having no gender at all [2]. Individuals with GD may undergo hormonal and surgical interventions in order to align their experienced and assigned genders, and/or may socially transition by changing their pronouns and appearance to that of their identified gender. Higher rates of GD have been found amongst autistic individuals than in the general population [86], and the rate of ASD traits and ASD within individuals with GD is also significantly higher than average [87]. This again suggests there may be shared factors between both conditions, and suggests populations in which to examine for ASD. The congruence between ASD and GD is of relevance to autistic females because some theories of gender differences in ASD emphasise high levels of androgen and other male hormones in autistic females, which may account for feelings of gender dysphoria amongst natal females but not males [88]. Individuals raised as female with higher than average androgen levels may therefore identify more with male peers and role models than female. It is especially important to identify the concordance between ASD and GD because the interventions for GD, especially when started before puberty, can have life-long consequences. ASD may impact how a person conceptualises gender and identity as well as their awareness of gender presentation [89], and so should be screened for and if necessary taken into account when planning GD interventions. 


\section{Future Perspective}

Current research into gender differences in ASD is focused around two main questions: are females generally protected from developing ASD in comparison to males, and do females express their ASD in different ways to males. The task for future research is to further test these two hypotheses, with the aim of better understanding the nature of the condition, and ensuring all autistic individuals receive the support and services that they require.

The current study of the genetics of broader populations of autistic individuals, focusing on those with and without intellectual disability, will shed more light on female protective genetic factors. Meanwhile, the blossoming area of research into camouflaging is just one way to explore the idea of different phenotypic expressions of ASD. A current issue is the use of potentially biased diagnostic criteria, meaning the true prevalence of female ASD cannot be estimated and therefore the Female Protective Effect hypothesis cannot be empirically tested. There is a vicious circle where females need greater severity to meet diagnostic criteria, and so may demonstrate a corresponding greater genetic load when compared to males. The development of more sensitive diagnostic criteria, including a greater range of behavioural presentations, is necessary in particular to identify those females with less severe impairments who may therefore also have lighter genetic loads, and are currently being missed.

Considering all the evidence for a protective genetic effect and a female phenotype of ASD, it is likely that both of these account for the relative under-representation of females in ASD: Females are less likely to develop ASD, but when they do they are also less likely to be identified. Future study will use the interaction between both of these effects to develop more sensitive diagnostic criteria, and gene-environment interaction studies may reveal how some of the genetic variation in those females who do have ASD may lead to different behavioural 
presentations. Longitudinal studies following autistic people of all genders will enable researchers and clinicians to identify factors promoting and preventing accurate, on-time diagnosis, and will provide both theoretical and practical information to make the lives of autistic individuals as meaningful and enjoyable as possible. Future research should follow the four levels of research themes suggested by Lai and colleagues in their conceptual framework [39], to ensure that it is addressing the most important questions and can be synthesised to current understanding of gender differences in ASD.

\section{Executive Summary}

\section{Gender differences in diagnosis}

- Autism Spectrum Disorders (ASD) are diagnosed more frequently in males than in females.

\section{The Female Protective Effect: Genetics}

- ASD has been shown to be highly heritable but with multiple genes associated with increased risk; no single 'autism gene' has been identified.

- Environmental effects, in particular gene-environment interactions, should be considered.

\section{Female Protective Effect}

- Females with ASD appear to have more genetic mutations than males with ASD; some studies also suggest that relatives of females with ASD are more likely to have ASD than relatives of males with ASD, due to a greater shared genetic risk.

Female presentation of ASD 
- Behavioural comparisons suggest few significant differences in males' and females' social and communication impairments, but that females with ASD may show fewer restricted and repetitive behaviours.

- Females may display a different pattern of associated ASD characteristics to males, meaning it is harder for females to receive a diagnosis using the male-based diagnostic criteria.

\section{Camouflaging by females}

- The external presentation of ASD may be camouflaged by some females, so the true extent of their impairments are not picked up by professional services.

\section{Missing Women: Co-occurring conditions and environmental fit}

- There most likely exists a group of females with ASD who are experiencing difficulties associated with their condition, but have not been able to access the support they need.

\section{Changing environments}

- The fit between an individual with ASD and their environment may change across the lifetime, such that difficulties which were previously compensated for or hidden come to light later on in life.

\section{Anorexia Nervosa}

- Similarities in cognitive biases between anorexia and ASD suggest that some women with anorexia may have an underlying ASD.

\section{Anxiety}


- Many women diagnosed with ASD in adulthood report experiencing anxiety problems prior to their diagnosis; this is therefore an important group to consider when assessing 'missed women'.

\section{Gender Dysphoria}

- The prevalence of gender dysphoria is significantly higher in ASD groups than in the general population, with implications for interventions and outcomes for both conditions.

\section{Financial \& Competing Interests Disclosure; Acknowledgements}

The authors have no relevant affiliations or financial involvement with any organization or entity with a financial interest in or financial conflict with the subject matter or materials discussed in the manuscript. This includes employment, consultancies, honoraria, stock ownership or options, expert testimony, grants or patents received or pending, or royalties. No writing assistance was utilized in the production of this manuscript. 


\section{References}

1. Kenny L, Hattersley C, Molins B, Buckley C, Povey C. \& Pellicano E. Which terms should be used to describe autism? Perspectives from the UK autism community. Autism, 20(4), 442-462 (2016).

2. American Psychiatric Association. DSM 5. American Psychiatric Association (2013).

3. Baio J. Prevalence of autism spectrum disorders--autism and developmental disabilities monitoring network, 14 sites, United States, 2010. MMWR. Surveillance Summaries : Morbidity and Mortality Weekly Report. Surveillance Summaries / CDC, 63, 1-21 (2014).

4. KimYS, Leventhal BL, Koh Y-J, Fombonne E, Laska E, Lim E-C, ... Grinker RR. Prevalence of autism spectrum disorders in a total population sample. Am. J. Psychiatry, 168(9), 904-12 (2011).

5. Blumberg SJ, Bramlett MD, Kogan MD, Schieve LA, Jones JR, \& Lu MC. Changes in prevalence of parent-reported autism spectrum disorder in school-aged U. S. children: 2007 to 2011 - 2012. Natl. Health Stat. Report, (65), 1-11 (2013).

6. Van Wijngaarden-Cremers PJM, van Eeten E, Groen WB, Van Deurzen PA, Oosterling IJ, \& Van der Gaag RJ. Gender and age differences in the core triad of impairments in autism spectrum disorders: a systematic review and meta-analysis. J. Autism Devl. Disord., 44(3), 627-35 (2014).

7. Loomes R, Hull L, \& Mandy WPL. What is the Male-to-Female Ratio in Autism Spectrum Disorder? A Systematic Review and Meta-Analysis. J. Am. Acad. Child Adolesc. Psychiatry, 56(6), 466-474 (2017).

8. Sun X, Allison C, Auyeung B, Baron-Cohen S, \& Brayne C. Parental concerns, socioeconomic status, and the risk of autism spectrum conditions in a population-based study. Res. Dev. Disabil., 35(12), 3678-3688 (2014).

9. Fombonne E. Epidemiology of pervasive developmental disorders. Pediatr. Res., 65(6), 591-8 (2009). 
10. Silberman S. Neurotribes: The legacy of autism and the future of neurodiversity. London, UK: Allen \& Unwin (2015).

11. Robinson EB, Lichtenstein P, Anckarsäter H, Happé F, \& Ronald A. Examining and interpreting the female protective effect against autistic behavior. Proc. Natl. Acad. Sci. U.S.A, 110(13), 5258-62 (2013).

12. Ronald A, \& Hoekstra RA. Autism spectrum disorders and autistic traits: a decade of new twin studies. Am. J. Med. Genet. B Neuropsychiatr. Genet., 156B(3), 255-74 (2011).

13. Tick B, Bolton P, Happé F, Rutter M, \& Rijsdijk F. Heritability of autism spectrum disorders: A meta-analysis of twin studies. J. Child Psychol. Psychiatry, 57(5), 585-595 (2016).

14. Gaugler T, Klei L, Sanders SJ, Bodea CA, Goldberg AP, Lee AB., ... Buxbaum JD. Most genetic risk for autism resides with common variation. Nat. Genet., 46(8), 881-5 (2014).

15. Sandin S, Lichtenstein P, Larsson H., Hultman, CM \& Reichenberg A. The familial risk of autism. JAMA, 311(17), 24794370 (2014).

16. Sato D, Lionel AC, Leblond CS, Prasad A, Pinto D, Walker S, ... Scherer SW. SHANK1 deletions in males with autism spectrum disorder. Am. J. Hum. Genet., 90(5), 879-887 (2012).

17. Vaags AK, Lionel AC, Sato D, Goodenberger M, Stein QP, Curran S, ... Scherer SW. Rare Deletions at the Neurexin 3 Locus in Autism Spectrum Disorder. Am. J. Hum. Genet., 90, 133-141 (2012).

18. Sanders SJ, Ercan-Sencicek AG, Hus V, Luo R, Murtha MT, Moreno-De-Luca D, ... State MW. Multiple Recurrent De Novo CNVs, Including Duplications of the 7q11.23 Williams Syndrome Region, Are Strongly Associated with Autism. Neuron, 70(5), 863885 (2011).

19. Sasson NJ, Lam KSL, Parlier M, Daniel J., \& Piven J. Autism and the broad autism phenotype: familial patterns and intergenerational transmission. J. Neurodev. Disord., 5(11), 40700 (2013). 
20. Devlin B, \& Scherer SW. Genetic architecture in autism spectrum disorder. Curr. Opin. Genet. Dev. 22(3), 229-237 (2012).

21. Betancur C. Etiological heterogeneity in autism spectrum disorders: More than 100 genetic and genomic disorders and still counting. Brain Research, 1380, $42-77$ (2011).

22. Chaste P. \& Leboyer M. Autism risk factors: Genes, environment, and gene-environment interactions. Dialogues Clin. Neurosci. 14(3), 281-292 (2012).

23. Mandy W, \& Lai M-C. Annual Research Review: The role of the environment in the developmental psychopathology of autism spectrum condition. J. Child Psychol. Psychiatry, 57(3), 271-92 (2016).

24. Hallmayer J, Cleveland S, Torres A, Phillips J, Cohen B, Torigoe T, ... Risch N. Genetic Heritability and Shared Environmental Factors Among Twin Pairs With Autism. Arch. Gen. Psychiatry, 68(11), 1095-1102 (2011).

25. Gilman SR, Iossifov I, Levy D, Ronemus M, Wigler M, \& Vitkup D. Rare De Novo Variants Associated with Autism Implicate a Large Functional Network of Genes Involved in Formation and Function of Synapses. Neuron, 70(5), 898-907 (2011).

26. Levy D, Ronemus M, Yamrom B, Lee Y-H, Leotta A, Kendall J, ... Wigler M. Rare De Novo and Transmitted Copy-Number Variation in Autistic Spectrum Disorders. Neuron, 70(5), 886-897 (2011).

27. Geier DA, Kern JK, King PG, Sykes LK, \& Geier MR. An evaluation of the role and treatment of elevated male hormones in autism spectrum disorders. Acta Neurobiol. Exp., 72(1), 1-17 (2012).

28. Rivet TT, \& Matson JL. Review of gender differences in core symptomatology in autism spectrum disorders. Res. Autism Spect. Disord., 23(3), 957-976 (2011).

29. Werling DM, \& Geschwind DH. Sex differences in autism spectrum disorders. Curr. Opin. Neurol. 26(2), 146-53 (2013). 
FEMALES WITH AUTISM SPECTRUM DISORDER

30. Desachy G, Croen LA, Torres AR, Kharrazi M, Delorenze GN, Windham GC, ... Weiss LA. Increased female autosomal burden of rare copy number variants in human populations and in autism families. Mol. Psychiatry, 20(2), 170-5 (2015).

31. Frazier TW, Youngstrom EA, Hardan AY, Georgiades S, Constantino JN, \& Eng C.

Quantitative autism symptom patterns recapitulate differential mechanisms of genetic transmission in single and multiple incidence families. Mol. Autism, 6, 58 (2015).

32. Szatmari P, Liu X-Q, Goldberg J, Zwaigenbaum L, Paterson AD, Woodbury-Smith M, ... Thompson A. Sex differences in repetitive stereotyped behaviors in autism: implications for genetic liability. Am. J. Med. Genet. B Neuropsychiatr. Genet., 159B(1), 5-12 (2012).

33. Ozonoff S, Young G, Carter A, Messinger D, Yirmiya N, Zwaigenbaum L, ... Hutman T. Recurrence risk for autism spectrum disorders: a Baby Siblings Research Consortium study. Pediatrics, 128(3), 488-495 (2011).

34. Harrington RA, Lee L-C, Crum RM, Zimmerman AW, \& Hertz-Picciotto I. Prenatal SSRI Use and Offspring With Autism Spectrum Disorder or Developmental Delay. Pediatrics, 133(5), e1241-e1248 (2014).

35. Baron-Cohen S, Auyeung B, Nørgaard-Pedersen B, Hougaard DM, Abdallah MW, Melgaard L, ... Lombardo MV. Elevated fetal steroidogenic activity in autism. Mol. Psychiatry, 20(3), 369-376 (2015).

36. Sandin S, Hultman CM, Kolevzon A, Gross R, MacCabe JH, \& Reichenberg A. Advancing maternal age is associated with increasing risk for autism: A review and meta-analysis. J. Am. Acad. Child Adolesc. Psychiatry, 51(5), 477-486.e1 (2012).

37. Kirkovski M, Enticott PG, \& Fitzgerald PB. A review of the role of female gender in autism spectrum disorders. J. Autism Devl. Disord., 43(11), 2584-603 (2013).

38. Gould J, \& Ashton-Smith J. Missed diagnosis or misdiagnosis? Girls and women on the autism spectrum. Good Autism Practice, 12(1), 34-41 (2011). 
FEMALES WITH AUTISM SPECTRUM DISORDER

39. Lai M-C, Baron-Cohen S, \& Buxbaum JD. Understanding autism in the light of sex/gender. Mol. Autism, 6(24), 1-5 (2015).

40. Hull L, Petrides KV, \& Mandy W. Behavioural and cognitive sex/gender differences in autism spectrum condition and typically developing males and females. Autism, Special Issue. (2016).

**41. Lai M-C, Lombardo MV, Auyeung B, Chakrabarti B, \& Baron-Cohen S. Sex/gender differences and autism: setting the scene for future research. J. Am. Acad. Child Adolesc. Psychiatry, 54(1), 11-24 (2015).

Proposes a framework of research themes to focus future research into sex/gender differences and autism: nosological and diagnostic challenges; sex/gender-independent and sex/gender dependent characteristics; general models of etiology; and specific etiological-developmental mechanisms.

42. Mandy W, Chilvers R, Chowdhury U, Salter G, Seigal A, \& Skuse D. Sex differences in autism spectrum disorder: evidence from a large sample of children and adolescents. $J$. Autism Devl. Disord., , 42(7), 1304-13 (2012).

43. Frazier TW, Georgiades S, Bishop SL, \& Hardan AY. Behavioral and cognitive characteristics of females and males with autism in the Simons Simplex Collection. $J$. Am. Acad. Child Adolesc. Psychiatry, 53(3), 329-40-3 (2014).

44. Park S, Cho SC, Cho IH, Kim B-N, Kim J-W, Shin M-S, ... Yoo HJ. Sex differences in children with autism spectrum disorders compared with their unaffected siblings and typically developing children. Res. Autism Spectr. Disord., 6(2), 861-870 (2012).

**45. Dworzynski K, Ronald A, Bolton P, \& Happé F. How different are girls and boys above and below the diagnostic threshold for autism spectrum disorders? J. Am. Acad. Child Adolesc. Psychiatry, 51(8), 788-97 (2012).

Introduces the concept of the female protective effect by demonstrating that females require a greater level of impairment to receive an ASD diagnosis than males, regardless of actual ASD traits 
46. Wilson CE, Murphy CM, McAlonan G, Robertson DM, Spain D, Hayward H, ... Murphy DG. Does sex influence the diagnostic evaluation of autism spectrum disorder in adults? Autism, 20(7), 808-819 (2016).

47. Beggiato A, Peyre H, Maruani A, Scheid I, Rastam M, Amsellem F, ... Delorme R. Gender differences in autism spectrum disorders: Divergence among specific core symptoms. Autism Research, 1-10 (2016).

48. Lord C, Risi S, Lambrecht L, Cook, EHC Jr, Leventhal BL, DiLavore PC, ... Rutter M. The Autism Diagnostic Observation Schedule-Generic: A Standard Measure of Social and Communication Deficits Associated with the Spectrum of Autism. J. Autism Devl. Disord., 30(3), 205-223 (2000).

49. Kreiser NL, \& White SW. ASD in females: are we overstating the gender difference in diagnosis? Clin. Child. Fam. Psychol., 17(1), 67-84 (2014).

50. Lai M-C, Lombardo MV, Pasco G, Ruigrok ANV, Wheelwright SJ, Sadek SA, ... BaronCohen S. A behavioral comparison of male and female adults with high functioning autism spectrum conditions. PloS One, 6(6), e20835 (2011).

51. Lemon JM, Gargaro B, Enticott PG, \& Rinehart NJ. Executive functioning in autism spectrum disorders: a gender comparison of response inhibition. J. Autism Devl. Disord., 41(3), 352-6 (2011).

52. Bölte S, Duketis E, Poustka F, \& Holtmann M. Sex differences in cognitive domains and their clinical correlates in higher-functioning autism spectrum disorders. Autism, 15(4), 497-511 (2011).

53. Lai M-C, Lombardo MV, Ruigrok ANV, Chakrabarti B, Wheelwright SJ, Auyeung B, ... Baron-Cohen S. Cognition in males and females with autism: similarities and differences. PloS One, 7(10), e47198 (2012).

54. Holt RJ, Chura LR, Lai M-C, Suckling J, von dem Hagen E, Calder AJ, ... Spencer MD. "Reading the Mind in the Eyes": an fMRI study of adolescents with autism and their siblings. Psychol. Med., 44(15), 3215-3227 (2014). 
55. Harrop C, Green J, \& Hudry K. Play complexity and toy engagement in preschoolers with autism spectrum disorder: Do girls and boys differ? Autism, 1-14 (2016).

56. Sedgewick F, Hill V, Yates R, Pickering L, \& Pellicano E. Gender Differences in the Social Motivation and Friendship Experiences of Autistic and Non-autistic Adolescents. J. Autism Devl. Disord., 46(4), 1297-1306 (2015).

57. May T, Cornish K, \& Rinehart NJ. Gender Profiles of Behavioral Attention in Children With Autism Spectrum Disorder. J. Atten. Disorders, 20(7), 627-635 (2012).

58. Oswald TM, Winter-Messiers MA, Gibson B, Schmidt AM, Herr CM, \& Solomon M. Sex Differences in Internalizing Problems During Adolescence in Autism Spectrum Disorder. J. Autism Devl. Disord., 46(2), 624-636 (2015).

59. Lai M-C, Lombardo MV, Ruigrok ANV, Chakrabarti B, Auyeung B, Szatmari P, ... MRC AIMS Consortium. Quantifying and exploring camouflaging in men and women with autism. Autism, (November), 1-13 (2016).

60. Lehnhardt F-G, Falter CM, Gawronski A, Pfeiffer K, Tepest R, Franklin J, \& Vogeley K. Sex-Related Cognitive Profile in Autism Spectrum Disorders Diagnosed Late in Life: Implications for the Female Autistic Phenotype. J. Autism Devl. Disord., 46(1), 139-154 (2015).

61. Bargiela S, Steward R, \& Mandy W. The Experiences of Late-diagnosed Women with Autism Spectrum Conditions: An Investigation of the Female Autism Phenotype. $J$. Autism Devl. Disord., Special Issue (2016).

62. Tierney S, Burn, J, \& Kilbey E. Looking behind the mask: Social coping strategies of girls on the autistic spectrum. Res. Autism Spectr. Disord., 23, 73-83 (2016).

63. Dean M, Harwood R, \& Kasari C. The art of camouflage: Gender differences in the social behaviors of girls and boys with autism spectrum disorder. Autism, (Special Issue), 1-12 (2016). 
64. Hull L, Petrides KV, Allison C, Smith P, Baron-Cohen S, Lai M-C, \& Mandy W. “ Putting on My Best Normal ": Social Camouflaging in Adults with Autism Spectrum Conditions. J. Autism Devl. Disord., 1-16 (2017).

65. Biederman J, Kwon A, Aleardi M, Chouinard VA, Marino T, Cole H, ... Faraone SV. Absence of gender effects on attention deficit hyperactivity disorder: findings in nonreferred subjects. Am. J. Psychiatry, 162(6), 1083-1089 (2005).

66. Biederman J, Mick E, Faraone SV, Braaten E, Doyle A, Spencer T, ... Johnson MA. Influence of gender on attention deficit hyperactivity disorder in children referred to a psychiatric clinic. Am. J. Psychiatry, 159(1), 36-42 (2002).

67. Begeer S, Mandell D, Wijnker-Holmes B, Venderbosch S, Rem D, Stekelenburg F, \& Koot HM. Sex Differences in the Timing of Identification Among Children and Adults with Autism Spectrum Disorders. J. Autism Devl. Disord., 43, 1151-1156 (2013).

**68. Lai M-C, \& Baron-Cohen SB. Identifying the lost generation of adults with autism spectrum conditions. Lancet Psychiatry, 2(11), 1013-1027 (2015).

Explores reasons why adults with autism and no intellectual disability may have failed to receive a diagnosis during childhood, and why it can be hard for them to receive a diagnosis in adulthood.

69. Rutherford M, McKenzie K, Johnson T, Catchpole C, O’Hare A, McClure I, ... Murray A Gender ratio in a clinical population sample, age of diagnosis and duration of assessment in children and adults with autism spectrum disorder. Autism (2016).

70. Happé FG, Mansour H, Barrett P, Brown T, Abbott P, \& Charlton RA. Demographic and Cognitive Profile of Individuals Seeking a Diagnosis of Autism Spectrum Disorder in Adulthood. J. Autism Devl. Disord., 46(11), 3469-3480 (2016).

71. Baldwin S, \& Costley D. The experiences and needs of female adults with highfunctioning autism spectrum disorder. Autism, 20(4), 483-495 (2015). 
72. Roberts AL, Koenen KC, Lyall K, Robinson EB, \& Weisskopf MG. Association of autistic traits in adulthood with childhood abuse, interpersonal victimization, and posttraumatic stress. Child Abuse Negl., 45, 135-42 (2015).

73. Buck TR, Viskochil J, Farley M, Coon H, McMahon WM, Morgan J, \& Bilder DA. Psychiatric Comorbidity and Medication Use in Adults with Autism Spectrum Disorder. J. Autism Devl. Disord., 44(12), 3063-3071 (2014).

74. Moss P, Howlin P, Savage S, Bolton P, \& Rutter M. Self and informant reports of mental health difficulties among adults with autism findings from a long-term follow-up study. Autism, 19(7) (2015).

75. Micali N, Hagberg KW, Petersen I, \& Treasure JL. The incidence of eating disorders in the UK in 2000-2009: findings from the General Practice Research Database. BMJ Open, 3(5), 3-10 (2013).

76. Westwood H, \& Tchanturia K. Autism Spectrum Disorder in Anorexia Nervosa: An Updated Literature Review. Curr. Psychiatry Rep., 19(7), 41 (2017).

77. Mansour, S., Rozenblat, V., Fuller-Tyszkiewicz, M., Paganini, C., Treasure, J., \& Krug, I. (2016). Emotions mediate the relationship between autistic traits and disordered eating: A new autistic-emotional model for eating pathology. Psychiatry Research, 245, 119126. http://doi.org/10.1016/j.psychres.2016.08.021

78. Tchanturia, K., Davies, H., Roberts, M., Harrison, A., Nakazato, M., Schmidt, U., ... Morris, R. (2012). Poor cognitive flexibility in eating disorders: Examining the evidence using the wisconsin card sorting task. PLoS ONE, 7(1). http://doi.org/10.1371/journal.pone.0028331

79. Pellicano L, \& Hiller RM. Anorexia and autism: A cautionary note. The Psychologist, 26, 780 (2013).

80. Mandy W, \& Tchanturia K. Do women with eating disorders who have social and flexibility difficulties really have autism? A case series. Mol. Autism, 6(1), 6 (2015). 
FEMALES WITH AUTISM SPECTRUM DISORDER

81. Westwood H, Eisler I, Mandy W, Leppanen J, Treasure J, \& Tchanturia K. Using the Autism-Spectrum Quotient to Measure Autistic Traits in Anorexia Nervosa: A Systematic Review and Meta-Analysis. J. Autism Devl. Disord., 46(3), 964-977 (2016).

82. Lugnegård T, Hallerbäck MU, \& Gillberg C. Psychiatric comorbidity in young adults with a clinical diagnosis of Asperger syndrome. Res. Dev. Disab., 32(5), 1910-7 (2011).

83. Hiller RM, Young RL, \& Weber N. Sex differences in autism spectrum disorder based on DSM-5 criteria: evidence from clinician and teacher reporting. $J A C P, 42(8), 1381-93$ (2014).

84. Solomon M, Miller M, Taylor SL, Hinshaw SP, \& Carter CS. Autism symptoms and internalizing psychopathology in girls and boys with autism spectrum disorders. $J$. Autism Devl. Disord., 42(1), 48-59 (2012).

85. Moss P, Howlin P, Savage S, Bolton P, \& Rutter M. Self and informant reports of mental health difficulties among adults with autism findings from a long-term follow-up study. Autism, 19(7) (2015).

86. Williams CVE. Diagnosing/Recognising High Functioning Autism in Adult Females: Challenging Stereotypes. Autism-Open Access, 6(3), 2-4 (2016).

87. Glidden D, Bouman WP, \& Jones BA. Gender Dysphoria and Autism Spectrum Disorder : A Systematic Review of the Literature. Sexual Medicine Review, 4(1), 3-14 (2016).

88. Kaltiala-Heino R, Sumia M, Työläjärvi M, \& Lindberg N. Two years of gender identity service for minors: overrepresentation of natal girls with severe problems in adolescent development. Child Adolesc. Psychiatry Ment. Health, 9, 9 (2015).

89. Jones RM, Wheelwright S, Farrell K, Martin E, Green R, Di Ceglie D, \& Baron-Cohen S. Brief report: Female-to-male transsexual people and autistic traits J. Autism Devl. Disord., 42(2), 301-306 (2012). 
90. Jacobs LA, Rachlin K, Erickson-Schroth L, \& Janssen A. Gender Dysphoria and CoOccurring Autism Spectrum Disorders: Review, Case Examples, and Treatment Considerations. LGBT Health, 1(4), 277-282 (2014). 